\title{
Obstructive sleep apnea syndrome in a patient with medulloblastoma
}

\author{
Glen Greenough, ${ }^{1}$ Michael Sateia, and Camilo E. Fadul \\ Sleep Disorders Center, Dartmouth Hitchcock Medical Center, Lebanon, NH 03756
}

\begin{abstract}
We present one adult patient with medulloblastoma who developed polysomnographically documented obstructive sleep apnea after posterior fossa surgery. The sleep apnea worsened in conjunction with clinical and imaging-confirmed neoplastic progression and clinically improved after craniospinal radiation therapy. Medulloblastoma or its surgical treatment has never before been implicated in a sleep-related breathing disorder. We discuss possible mechanisms for its occurrence and management implications. Neuro-Oncology 1, 289-291, 1999 (Posted to Neuro-Oncology [serial online], Doc. 99-08, September 9, 1999. URL <neuro-oncology.mc.duke.edu>)
\end{abstract}

$\mathrm{O}$ bstructive sleep apnea results from obstruction of the upper airway during sleep. Upper airway obstruction may develop secondary to nasal obstruction, enlarged upper airway structures, jaw abnormalities, adipose deposition in the upper airway, obesity, or any disorder that impairs the maintenance of muscular tone or control of the pharynx. This obstruction leads to desaturation and increasing respiratory effort. The increase in effort eventually leads to arousal and resumption of air flow. This cycle repeats itself thereby fragmenting sleep. Obstructive sleep apnea has been associated with multiple neurologic conditions including stroke and various degenerative diseases. Nonneoplastic lesions in the posterior fossa, such as syringobulbia and olivopontocerebellar degeneration, have been reported to cause obstructive sleep apnea (Adelman et al., 1984). In our review of the literature, we could find only one reference to a CNS neoplasm causing obstructive sleep apnea, and it involved a lipoma of the mesencephalic tectum and ros-

Received 2 February 1999, accepted 23 June 1999.

${ }^{1}$ Address correspondence and reprint requests to Glen Greenough, M.D., Sleep Disorders Center, Dartmouth Hitchcock Medical Center, One Medical Center Drive, Lebanon, NH 03756.

${ }^{2}$ Abbreviation used is as follows: CPAP, continuous positive airway pressure. tral pons in a patient who had mixed sleep apneas (Sheridan et al., 1990). The remainder of the literature on CNS neoplasms and sleep apnea consists of examples of central sleep apnea. Ito et al. (1996) describe two pediatric patients with brainstem gliomas, one of whom had documented central sleep apneas. There is one case of nocturnal desaturations without snoring in a patient with a sphenoidal meningioma (Garcia-Rio et al., 1997). The lack of snoring suggests a central etiology, but polysomnography was not done to confirm the type of breathing disturbance. We describe a patient with medulloblastoma, a primitive neuroectodermal tumor localized to the posterior fossa, who developed symptomatic obstructive sleep apnea syndrome.

\section{Case Study}

A 34-year-old man with a past medical history notable only for tonsillectomy and adenoidectomy as a child developed vomiting and vertigo followed by headache in August 1997. He had no history of snoring, apneas, or sleeping difficulties. Two months after the onset of symptoms, he had an MRI of the brain that disclosed a bilateral cerebellar hemispheric lesion. At the preoperative physical exam, the patient weighed $87 \mathrm{~kg}$ and had a body mass index of 27.5. The patient was started on dexamethasone, which was continued throughout the described clinical course at varying doses. The patient had been on the dexamethasone only days before the operation. A neoplasm, later pathologically identified as a medulloblastoma, was removed via a suboccipital craniotomy in October 1997. One hour after the procedure, the patient developed a left cerebellar hemorrhage requiring hematoma evacuation. Postoperatively, he required minimal ventilatory assistance, but needed an endotracheal tube to maintain airway patency. A tracheostomy was performed because of repeated failed extubations secondary to airway obstruction. Five days after the craniotomy, the patient returned to the operating room for bilateral cerebellar debridement and placement of a ven- 
tricular drain because of a recurrence of the hemorrhage. His weight at this time was down to $76 \mathrm{~kg}$. The patient continued to have irregular breathing and no purposeful motor activity after extubation. Gradually he improved, so that by six weeks after the craniotomy he was awake, moving all four extremities to command, and able to communicate when his tracheostomy was plugged. A follow-up MRI in early December revealed subacute blood in the left cerebellar hemisphere but no hydrocephalus. The medulla and pons were mildly shifted to the right. He was discharged to a rehabilitation facility in December 1997, and within a week his tracheostomy had been plugged permanently. Further treatment of his medulloblastoma was deferred until it was determined that the patient could tolerate it. The patient's weight two weeks prior to the plugging of the tracheostomy was $77 \mathrm{~kg}$, down $10 \mathrm{~kg}$ from admission.

When his tracheostomy was closed, the patient's family noted the gradual development of nocturnal snoring and apneas. On a nightly basis, the patient was observed to snore, stop breathing, and then awaken with a gasp or snort. He would seem to arouse and cough but then rapidly go back to sleep only to resume the cycle. Daytime somnolence and frequent coughing were also noted. The patient increased his sleep time each night to $12 \mathrm{~h}$, yet still required a 2- to 3-h nap during the day.

At the postoperative check in February 1998, he had greatly improved from a neurologic standpoint. He was alert and communicative, but with scanning speech, severe left-sided ataxia, and a partial left sixth nerve palsy. His snoring and sleep apneas continued despite his improvement in other ways. Over the next two months, the patient, however, began to decline neurologically with an accompanying worsening of his snoring, gasping, and nocturnal choking. In April 1998 the exam showed him to be alert but with severe dysarthria, scanning speech, bilateral dysmetria, truncal ataxia, and bilateral partial sixth nerve palsies. The gag reflex was hyperactive, but palate elevation and tongue protrusion were normal. By this time, the patient had also developed great difficulty swallowing the pureed diet he had been on for the last few months. Tube feedings were resumed. A repeat MRI was performed, and the tumor was noted to have recurred in the right cerebellar hemisphere. The brainstem was also shown to be significantly compressed on the right more than the left from the junction of the midbrain and pons down through the medulla. Shortly thereafter, craniospinal radiation was instituted.

In May 1998, the patient was admitted for difficulty with breathing. On the morning of admission, just after awakening, he had a pulse oximetry reading of $87 \%$ at rest. After a pulmonary evaluation, which included a normal chest radiograph, a low probability ventilation perfusion scan, and pulmonary function tests with a restrictive pattern, polysomnography was performed. The polysomnogram revealed that, after an initial 60min period of restlessness, the patient entered a more sustained period of sleep. He had an elevated percentage of stage 1 sleep at $20 \%$, but a reduced percentage of rapideye-movement sleep at $7 \%$. He manifested a high frequency of obstructive apneas and hypopneas. The apnea and hypopnea index was elevated at 81 events per hour (rapid-eye-movement apnea and hypopnea index of 96 events per hour). No central or mixed hypopneas or apneas were noted. The patient had frequent desaturations into the low 80 s by pulse oximetry. Some desaturations into the mid-70s were noted in rapid-eye-movement sleep. A few nights later, a CPAP ${ }^{2}$ titration was done with improvement of the apnea and hypopnea index to 3.6 events per hour on a pressure of $14 \mathrm{~cm}$ of water. Given his tolerance of and good response to CPAP, an otolaryngology evaluation was not undertaken.

The patient continued with radiation for a total of six weeks. He gradually improved to the point were he was able to leave the rehabilitation facility and go home three weeks after completing the radiation treatments. The CPAP reportedly significantly improved his snoring and apneas while at the rehabilitation hospital. He was doing so well that when he arrived home he stopped using the CPAP without consulting his physicians and had a mild recurrence of the apneas. The symptoms of obstructive sleep apnea, including daytime sleepiness, had significantly improved. Given the persistence of some symptoms, CPAP was eventually restarted with an empiric decrease in his pressure to $10 \mathrm{~cm}$ of water to improve compliance. A modified barium swallow in June 1998 revealed only a delayed swallow with premature spillage in the oral phase. For the remainder of the study, adequate lateralization of the bolus was seen without laryngeal penetration or nasopharyngeal reflux. A pureed diet was resumed. Follow-up scan in September 1998 revealed resolution of the lower brainstem compression with only residual left midbrain compression. The patient's weight at this time was $81 \mathrm{~kg}$, which was $6 \mathrm{~kg}$ less than his preoperative weight, but $4 \mathrm{~kg}$ more than his weight around the time the observed apneas were first noted. When the patient was interviewed in December 1998, he reported good compliance and no daytime sleepiness or snoring. He was sleeping $6-8 \mathrm{~h}$ a night with a scheduled 1-h nap each day. A follow-up sleep study was not obtained because of travel issues.

\section{Discussion}

The temporal course of this patient's sleep apnea strongly suggests a linkage of the medulloblastoma and the obstructive sleep apnea. The evolution of his sleep disruption seemed to parallel his clinical course. He had no evidence for obstructive sleep apnea prior to the development of his tumor and its treatment. He developed snoring and observed apneas postoperatively after closure of his tracheostomy. As his tumor recurred, his apneas appeared to worsen. After treatment with radiation, his apneas and snoring improved to the point where he stopped using his CPAP. Unfortunately, we were unable to obtain repeat polysomnography to document improvement after the radiation treatment. The parallel course of his swallowing difficulties supports the causative link between his medulloblastoma and his sleep apnea. Although this course suggests that the patient's medulloblastoma was the cause of his obstructive sleep apnea, the patient's surgery probably played a role in its initial development. Adelman et al. (1984) report the 
development of obstructive sleep apnea after neurosurgical decompression of a cervical syrinx associated with syringobulbia. If the surgery, however, were the only factor responsible for his symptoms, we would not expect his apnea to be worse as his tumor recurred and to subjectively improve with radiation treatment. It is unlikely that the patient's weight changes played a significant role in development of his sleep apnea given that his weight, when he was noted to have improved in September 1998, was $4 \mathrm{~kg}$ more than his weight when the sleep apnea was first clinically noted in December 1997.

There is a theoretical risk that a posterior fossa tumor could cause sleep apnea. Damage to the medulla has been shown to produce central sleep apnea (Auer et al., 1996; Ito et al., 1996). Central sleep apnea may result from damage to the medullary nuclear groups, such as the nucleus tractus solitarius or nucleus ambiguus, which play a role in shaping respiratory output. This patient, however, developed obstructive and not central sleep apnea. The only precedent for this is the report (Sheridan et al., 1990) of one patient with a lipoma of the mesencephalic tectum who developed mixed sleep apneas. At autopsy in that case, the patient's lipoma had infiltrated the right locus ceruleus and disrupted the normal tegmental architecture. During his decline, our patient's brain MRI five months postoperatively revealed compression of the brainstem on the right, more than the left, from the midbrain-pontine junction down through the medulla. Brainstem compression could lead to obstructive sleep apnea by damage to the locus ceruleus as Sheridan et al. (1990) postulate, or by disruption of the descending motor fibers, which could then lead to decreased pharyngeal tone and subsequent obstructive apneas. A third possibility is that direct involvement of cranial nerves 10 and 12, through tumor spread, could lead to decreased pharyngeal tone and obstructive sleep apnea on that basis. The meningeal enhancement noted on the MRI could reflect tumor spread through the meninges, which could disrupt cranial nerve function. The increased gag reflex in this patient, however, suggests an upper motor neuron and not a lower motor neuron problem. Degenerative disorders, such as multiple system atrophy, which involve the pons and cerebellum, have been implicated in both central (Salazar-Grueso et al., 1988) and obstructive sleep apnea (Adelman et al., 1984). The only case of multiple system atrophy with purely obstructive sleep apnea (Sadaoka et al., 1996), however, was also associated with fixation of the right vocal fold in the paramedian position. Isolated cerebellar damage has never been shown to lead to purely obstructive sleep apnea, but one might imagine that it could on the basis of hypotonia.

Despite the probable central origin of this patient's obstructive sleep apnea, he responded well to treatment with CPAP. His apnea and hypopnea index dramatically improved from 81.0 to 3.6 events per hour with the initiation of CPAP. This corresponded to a clinical improvement as well.

We report a case of a medulloblastoma, an infratentorial tumor, which led to the development or at least the exacerbation of an obstructive sleep apnea syndrome. The mechanism by which this tumor led to obstructive sleep apnea is unclear. The development of purely obstructive sleep apnea in a patient with only CNS disease raises many questions about the nature of the obstructive sleep apnea syndrome in general. CNS tumors have only rarely been implicated in sleep apnea. This case establishes a link between the obstructive sleep apnea syndrome and medulloblastoma in an adult. The appropriateness of generalizing this association to other patients with posterior fossa tumors remains to be established. To establish the prevalence of treatable obstructive sleep apnea in this population of patients, physicians will need to evaluate patients with posterior fossa tumors for the signs and symptoms of this syndrome. We report this case to raise the awareness of clinicians to the possibility of sleep apnea in patients with posterior fossa tumors. Treatment of sleep apnea can significantly improve the quality of a patient's life, making investigation of the possibility worthwhile, even when the longterm prognosis is grim.

\section{References}

Adelman, S., Dinner, D.S., Goren, H., Little, J., and Nickerson, P. (1984) Obstructive sleep apnea in association with posterior fossa neurologic disease. Arch. Neurol. 41, 509-510.

Auer, R.N., Rowlands, C.G., Perry, S.F., and Remmers, J.E. (1996) Multiple sclerosis with medullary plaques and fatal sleep apnea (Ondine's curse). Clin. Neuropathol. 15, 101-105.

Garcia-Rio, F., Prados, C., Racionero, M.A., Soledad, S., Pino, J.M., and Villamor, J. (1997) Nocturnal arterial oxygen desaturation secondary to a sphenoidal meningioma. Respiration 64, 229-232.

Ito, K., Murofushi, T., Mizuno, M., and Semba, T. (1996) Pediatric brain stem gliomas with the predominant symptom of sleep apnea. Int. J. Pediatr. Otorhinolaryngol. 37, 53-64.

Sadaoka, T., Kakitsuba, N., Fujiwara, Y., Kanai, R., and Takahashi, H. (1996) Sleep-related breathing disorders in patients with multiple system atrophy and vocal fold palsy. Sleep 19, 479-484.

Salazar-Grueso, E.F., Rosenberg, R.S., and Roos, R.P. (1988) Sleep apnea in olivopontocerebellardegeneration: Treatment with trazodone. Ann. Neurol. 23, 399-401.

Sheridan, F., Scharf, D., Henderson, V.W., and Miller, C.A. (1990) Lipomas of the mesancephalic tectum and rostral pons associated with sleep apnea syndrome. Clin. Neuropathol. 9, 152-156. 